\title{
Correlation of SMNt and SMNc gene copy number with age of onset and survival in spinal muscular atrophy
}

\author{
Joanne E Taylor ${ }^{1,4}$, Neil H Thomas ${ }^{2}$, Cathryn M Lewis' ${ }^{1}$, Stephen J A bbs ${ }^{1}$, \\ Nanda R R odrigues ${ }^{3}$, Kay E Davies ${ }^{3}$ and Christopher G M athew ${ }^{1}$ \\ ${ }^{1}$ D ivision of Medical and Molecular G enetics, United M edical and Dental Schools of G uy's and St Thomas' \\ H ospitals, G uy's Campus, L ondon \\ ${ }^{2} \mathrm{D}$ epartment of $\mathrm{C}$ hild $\mathrm{H}$ ealth, Southampton $\mathrm{G}$ eneral $\mathrm{H}$ ospital, Southampton \\ ${ }^{3} G$ enetics $L$ aboratory, D epartment of Biochemistry, University of $O$ xford \\ ${ }^{4} \mathrm{O}$ xford M edical G enetics L aboratories, Churchill H ospital, H eadington, O xford, UK
}

\begin{abstract}
Childhood-onset autosomal recessive spinal muscular atrophy (SMA) is associated with absence of the telomeric survival motor neuron gene (SMNt) in most patients, and deletion of the neuronal apoptosis inhibitory protein (NAIP) gene in the majority of severely affected patients. Analysis of SMNt has been complicated by the existence of a centromeric copy, SMNc, which is almost identical to SMNt but which can be distinguished from it by restriction enzyme analysis. In this study 143 SMA patients have been genotyped for the presence or absence of the SMNt, SMNc and NAIP genes, and the data correlated with quantifiable clinical variables. Although a significant correlation was observed between the presence or absence of the NAIP gene and the severity of the clinical phenotype in SMA patients generally, there was no difference in age of onset or survival in type I patients with the NAIP+ or NAIP- genotype. Fluorimetric PCR analysis of SMNc gene dosage in 57 patients homozygous for the absence of the SMNt gene but in whom the NAIP gene was present showed a highly significant correlation between SMNC copy number and SMA subtype, and between SMNc copy number and both age of onset and length of survival. The data provide strong statistical support for the emerging consensus that the clinical phenotype in SMA is directed primarily by the level of functional SMN protein. The lower SMNc copy number in type I patients in whom the NAIP gene is present suggests that the SMNt gene is removed by deletion in the majority of such patients, rather than by gene conversion as is the case in SMA types II and III.
\end{abstract}

Keywords: spinal muscular atrophy; survival motor neuron genes; gene dosage

\footnotetext{
Correspondence: D r Christopher M athew, D ivision of M edical \& M olecular Genetics UMDS, 8th Floor Guy's Tower, G uy's H ospital, L ondon SE 1 9RT, U K. Tel: +44 1719554653 Fax: +44 1719554644 E-mail: c.mathew@umds.ac.uk Received 20 November 1997; revised 17 February 1998; accepted 25 February 1998
}

\section{Introduction}

Childhood-onset proximal spinal muscular atrophy (SMA) is the second most common lethal autosomal recessive disorder after cystic fibrosis, affecting approximately 1 in 10000 births, with an approximate carrier frequency of 1 in 50 . It is a neurodegenerative disease 
which affects the $\alpha$ motor neurons of the spinal cord, leading to wasting of the proximal muscles and death, usually due to respiratory infection. The disorder has been classified into three forms, types I (WerdnigH offmann disease; severe SM A ; acute infantile SM A), II (intermediate) and III (Kugelberg-Welander disease; mild SM A ), which are defined by the age of onset and the clinical course of the disease.

A II three forms of SMA were mapped to the long arm of chromosome 5, at 5q11.2-13.3, ${ }^{1-3}$ indicating they could be allelic variants of the same disease. The analysis of multicopy polymorphic markers in the SM A candidate region provided the first indication that large-scale deletions could be involved in SM A. ${ }^{4}$ This work showed both inherited and de novo deletions in nine unrelated SMA patients and marked heterozygosity deficiency for the loci studied in at least $18 \%$ of SM A type I patients.

Two candidate genes for all three types of SM A were identified in 1995, named the survival motor neuron (SMN) gene $e^{5}$ and the neuronal apoptosis inhibitory protein (NAIP) gene. ${ }^{6}$ Both genes were found to be duplicated within the candidate region for SM A. The NAIP gene was found to be homozygously deleted in $27.3 \%$ of SMA patients tested, and homozygous deletions of NA IP were also observed in $1.8 \%$ of SM A carriers. ${ }^{6}$ The SM N gene exists in two almost identical forms, termed SMNt (telomeric) and SMNc (centromeric), which complicates the analysis of this gene. Both SM Nt and SM Nc encompass $20 \mathrm{~kb}$ of genomic DNA and have 9 exons. Sequence analysis has revealed only five single base differences between the two genes, one of which is in exon 7 and one in exon 8. PCR amplification and single-strand conformational polymorphism (SSCP) analysis allow a distinction to be made between exons 7 and 8 of the SM Nt and SM NC genes. This technique was used to demonstrate that $98.6 \%$ of SMA patients studied had homozygous deletions of the SMNt gene. ${ }^{5}$ No SMA carriers or normal control individuals were found to have homozygous deletions of the SM Nt gene, although homozygous deletions of the SM NC gene were observed in these groups.

These results indicated that the SM N t gene was more likely to have a role in the pathogenesis of SMA, particularly since those SM A patients without a homozygous deletion of the SM Nt gene were found to have a heterozygous deletion in conjunction with a point mutation in their intact copy of the SM Nt gene. Later studies identifying various small mutations in the $\mathrm{SM} \mathrm{N} \mathrm{t}$ gene provided further evidence that it was likely to be the primary SMA-determining gene. ${ }^{7-11}$ A dditional work with the multicopy markers C212 and C272 showed an association of specific numbers of marker copies with the severity of the disease, and a model was proposed to account for the different phenotypes observed by a system of mild and severe alleles. ${ }^{12}$ H owever, later studies ${ }^{13-15}$ described homozygous deletions of the SM N t gene in a small number of unaffected individuals (siblings and parents of affected individuals), thus casting some doubt upon this gene as the only causal gene for SM A. The case for at least partial involvement of the NAIP gene in the SMA phenotype was supported by several studies which demonstrated that the severe SM A type I was associated with a higher frequency of homozygous deletions of NAIP than the milder forms ${ }^{16-18} \mathrm{~A}$ study by Somerville et $\mathrm{al}^{19}$ quoted a five-fold increased risk of type I SMA associated with absence of the NAIP gene.

A further complication in the molecular pathology of SM A emerged with the finding that some SM A patients who were apparently homozygously deleted for exon 7 of SM Nt, but not for exon 8, had a chimeric gene in which exon 7 of SMNc was fused to exon 8 of SM Nt, probably as a result of gene conversion. ${ }^{5,7,12,20,21}$ Very recently, analysis of patients with SM A types II and III by pulsed-field gel electrophoresis showed physical evidence that some mutations previously classed as deletions actually resulted from gene conversions in which SM Nt was replaced by SM Nc. ${ }^{22}$ These observations suggested a possible explanation for the different clinical phenotypes seen in patients who appear to have the same genotype, since the SSCP and restriction digestion assays do not distinguish between the absence of SMNt as a result of gene deletions or of gene conversions. ${ }^{22}$ Thus if the loss of SM N tin SM A types II and III were the result of gene conversion rather than deletions, these patients would be left with a larger number of copies of SMNC than patients with SMA type I who had lost SM Nt as a result of genuine gene deletions. The difference in SMN gene copy number would also be reflected in a different amount of functional protein, since the SM N c gene is known to be transcribed. This hypothesis is supported by the recent demonstration of a correlation between disease severity and SM N protein levels in the liver and spinal cord of SMA patients, ${ }^{23}$ and by the finding of a higher ratio of SM N C/SM Nt gene dosage in the parents of SM A II and III patients compared with the parents of type I patients. ${ }^{16}$ A dditionally, Coovert et $\mathrm{al}^{24}$ reported a 
correlation between disease severity and the amount of nuclear SM N protein, located in structures called gems, and postulated that gem formation was the key to adequate function of the SM N protein.

This study investigates the clinical and molecular characteristics of 143 SM A patients in order to establish their genotype at the SM N/NA IP Ioci, and to relate this information to measurable clinical parameters such as age of onset and survival. A fluorimetric PCR assay has also been developed to measure dosage of the SMNC gene, and demonstrates a highly significant correlation between SMNC copy number and clinical severity in SM A.

\section{Materials and Methods}

\section{Patients}

The patients investigated in this study were referred to the South Thames ( $E$ ast) R egional G enetics Centre by clinicians from the $U$ nited $K$ ingdom and continental $E$ urope. D iagnosis rested with the individual clinicians, but review of referral details by one of us (NHT) has established that the index cases met the diagnostic criteria of the International Consortium on SM A ${ }^{25,26}$ both in terms of clinical features and diagnostic investigations. Samples were accompanied by a standard referral form listing inclusion and exclusion criteria, and only those patients meeting these criteria were included in the study. DNA was available from 143 SMA patients, comprising 97 type I, 38 type II and eight type III individuals.

\section{Assays}

Absence of the SMNt Gene Homozygous deletion or absence of exons 7 and 8 of the SM Nt gene was detected by restriction enzyme digestion assays based on the method published by van der Steege et al, ${ }^{27}$ but with internal controls for digestion incorporated.

NAIP Exon 5 of the NAIP gene was detected using the published biplex PCR assay which includes exon 13 of the NAIP gene as an internal control. ${ }^{6}$

SMN Dosage Assay A fluorescent assay, based on a method by $Y$ au et al $^{28}$ was developed to measure the ratio of SMN gene copy number (SMNt $+\mathrm{SMNC}$ ) relative to a control locus, the myelin protein zero (MPZ) gene. The forward primers R 111 and $541 C 960^{5}$ were end-labelled with FA M (blue) and used with the unlabelled reverse primers $541 C 770$ and $541 \mathrm{C} 1120$ to amplify SM N exons 7 and 8. Exons 1 and 3 of the M PZ gene were co-amplified with the SM N loci using similarly labelled primers for these exons. ${ }^{29}$ The amplification mixture contained: R 111, 541C 770, 541C 960 and $541 \mathrm{C} 1120$ at a concentration of $0.8 \mu \mathrm{M}, \mathrm{MPZ1F}, \mathrm{MPZ} 1 \mathrm{R}$, MPZ3F and MPZ3R at a concentration of $0.4 \mu \mathrm{M}, 500 \mu \mathrm{M}$ dNTPs, $1.5 \mathrm{~mm} \mathrm{M} \mathrm{gCl}_{2}, 1.5$ units Taq polymerase (Promega, Southampton) in a $25 \mu$ l reaction, using 'hot start'. The cycling times were as follows: 18 cycles of denaturation at $94^{\circ} \mathrm{C}$ for $20 \mathrm{~s}$, annealing at $51.5^{\circ} \mathrm{C}$ for $20 \mathrm{~s}$, extension at $70^{\circ} \mathrm{C}$ for $1 \mathrm{~min}$, using the Techne Progene thermal cycler. PCR product $(2.5 \mu \mathrm{l})$ was mixed with $3 \mu \mathrm{l}$ of formamide loading dye and $0.5 \mu \mathrm{l}$ of ROX-labelled size standard GS-500 and electrophoresed on an A BI 373A Genescanner (Perkin-E Imer/A BI, San Francisco, CA ). The data were transferred to G enotyper software and the ratio of SMN to MPZ was calculated by dividing the sum of the peak areas for SM N exons 7 and 8 by the sum of the peak areas for MPZ exons 1 and 3 .

\section{Genotyping}

The cohort of 143 patients with an unequivocal clinical diagnosis of SMA was typed with the SM Nt exons 7 and 8 assays and with the NAIP exon 5 assay, in order to establish the frequencies of homozygous deletions or absence of these two genes in SMA patients. DNA from 200 SMA carriers, who were all parents of affected children, and a group of 100 unaffected controls, who were all adults referred for reasons other than neuromuscular di-sease, was also typed with these assays.

A $n$ alternative approach to evaluating the data is to classify the patients by their genotype rather than on the basis of their clinical classification as types I-III, and then to assess the clinical phenotype which is associated with a particular genotype, using measurable clinical parameters. In order to examine the relationship between SM N t and NA IP genotypes and clinical features, the patients were then divided into genotypic groups on the basis of these results, as follows:

- Group $1(n=64)$ : SM Nt exons 7 and 8 and NAIP exon 5 all homozygously absent (- - -);

- Group 2 ( $n=64)$ : SM Nt exons 7 and 8 homozygously absent, at least one copy of NAIP exon 5 present $(--+)$;

- Group 3 ( $n=7$ ): SM N t exon 7 absent, at least one copy of exon 8 and NAIP exon 5 present $(-++)$;

- Group $4(n=8)$ : A t least one copy of SM Nt exons 7 and 8 and NAIP exon 5 all present $(+++)$.

R elevant clinical data, including age of onset, age of death and whether motor milestones (sitting and standing) were achieved, were available for these patients, although complete data for all features were not available for all patients. A statistical comparison of groups 1 and 2 was carried out, since sufficient numbers of patients had been studied.

A total of 57 SM A patients, all in genotype group $2(--+)$, was typed with the SM N dosage assay. Since they were known to have no SM N t product, this assay allowed an assessment of the relative numbers of copies of SM N c present. The patients comprised 26 type I, 23 type II and eight type III individuals. The assay was also used to type 27 unaffected control individuals in whom both SM Nt and SM Nc were present.

\section{Statistical Analyses}

For genotypic groups 1 and 2 (64 patients in each group), the mean ages of onset and death and the proportion of patients who achieved sitting and standing were calculated, and statistical analyses were performed. A histogram was produced showing the ages of onset for individuals in groups 1 and 2 , and the difference in onset age between the two groups was assessed using the non-parametric Wilcoxon rank sum test. The differences in the proportion of individuals in these groups who achieved sitting and standing were assessed using a t-test. 
$M$ any patients were still alive at the most recent follow-up, and in order to make use of this censored information, survival time rather than age of death was examined. Survival was modelled using a Kaplan-M eier survival curve, which takes into account observations from the individuals known still to be alive at a certain age.

The SM N:MPZ ratios were calculated for each patient in genotypic group 2, and these were subdivided depending on the clinical classification of the disease severity. A nalysis of variance was used to determine whether the SM N :M PZ ratios had a significant effect on the clinical subgroup. The effect of SM N :M PZ ratios on age at diagnosis and on survival was also investigated.

\section{Results}

The results of the analysis of SMNt and NAIP genotypes for 143 SM A patients, 200 SM A carriers and 100 unaffected controls were similar to previous studies, with homozygous absence of the SMNt gene in $94.4 \%$ of SMA patients tested, both exons 7 and 8 absent in $89.5 \%$, and exon 7 alone absent in a further $4.9 \%$ of patients. The $(-++)$ genotype was only seen in patients with SMA type II. A t least one copy of the SMNt gene was present in all SMA carriers and controls tested. No SMA patients had homozygous absence of the SM N c gene, but it was absent in $11 \%$ of controls. Exon 5 of the NA IP gene was absent in $44.8 \%$ of SM A patients and $4 \%$ of SM A carriers. The (-- - ) genotype was seen in $63.9 \%$ of SM A type I patients, but in only $5.3 \%$ of type II and no type III patients studied. The data confirm the high frequency of apparent deletions of the SM Nt gene in all classes of SM A, and the association of NAIP gene deletions with the more severe phenotype, in agreement with previous studies. $^{5,16-19}$

Figure 1 shows a histogram representing the distribution of ages of onset in groups 1 and $2(---$ and --+ genotypes respectively). Where both SM Nt and NAIP are absent, onset occurs earlier, and all patients with this genotype are clearly affected before the age of 10 months (mean $=2.16$ months). By contrast, in patients where NAIP is present, onset occurs significantly later $(P<0.0001)$ with a mean of 5.8 months, and $18 \%$ of patients are diagnosed between 1 and 2 years. The proportion of patients in groups 1 and 2 who achieved the clinical milestones of sitting and standing was $2.9 \%$ versus $49.2 \%$ and $0 \%$ versus $16.1 \%$, respectively $(P<0.0005$ in each case). The mean age of death in group 2 was higher than in group 1 - 30.36 months compared with 8.93 months. However, this figure is distorted by one outlier in group 2 - a type II patient who survived until the age of 39 years. The survival curve for these two groups (Figure 2) clearly shows that patients with the --+) genotype have a prolonged survival time compared with those with the (---) genotype. The median survival time, beyond which $50 \%$ of patients are still alive, was 7 months in the (---) genotype group, compared with 72 months in the $(--+)$ genotype group. Ten patients in group 2 were still alive after 10 years.
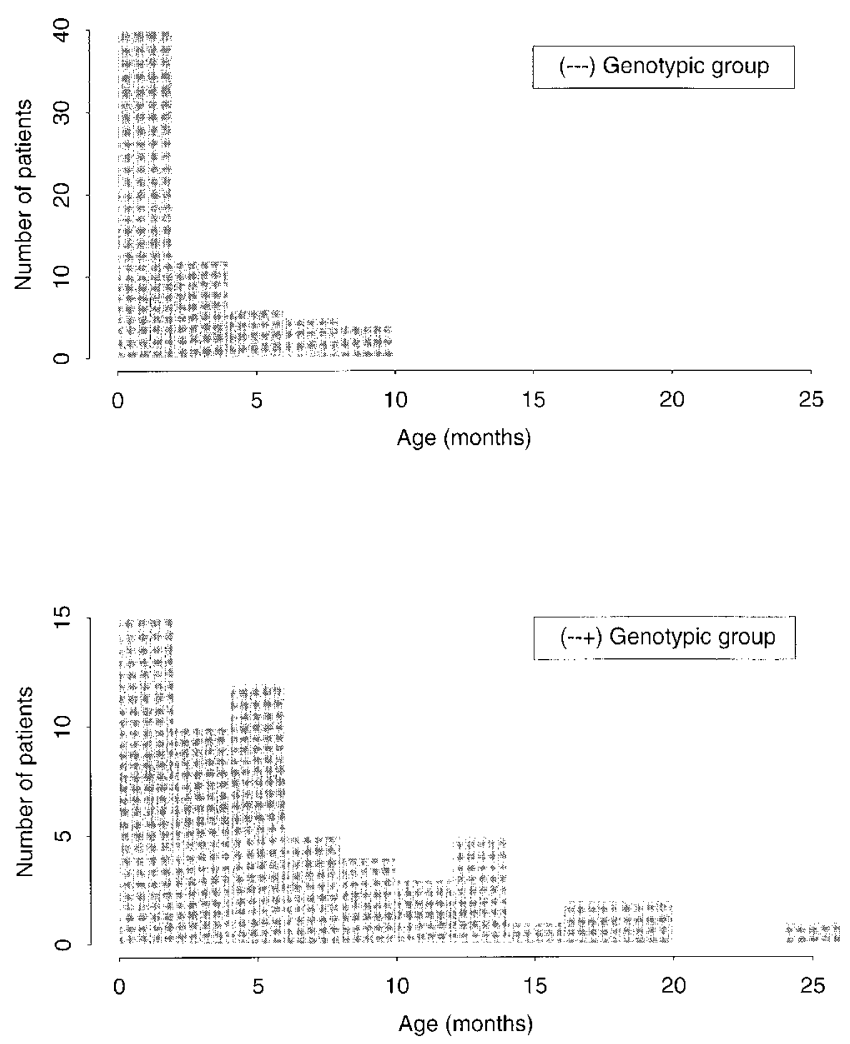

Figure $1 \mathrm{H}$ istograms showing earlier age of onset with absence of NAIP

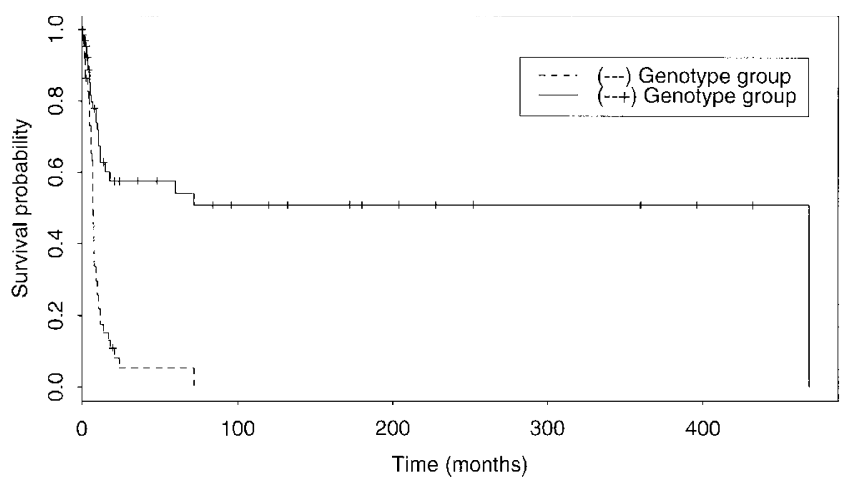

Figure 2 Survival curves showing significantly reduced survival with absence of NAIP. The short vertical lines each represent one individual 


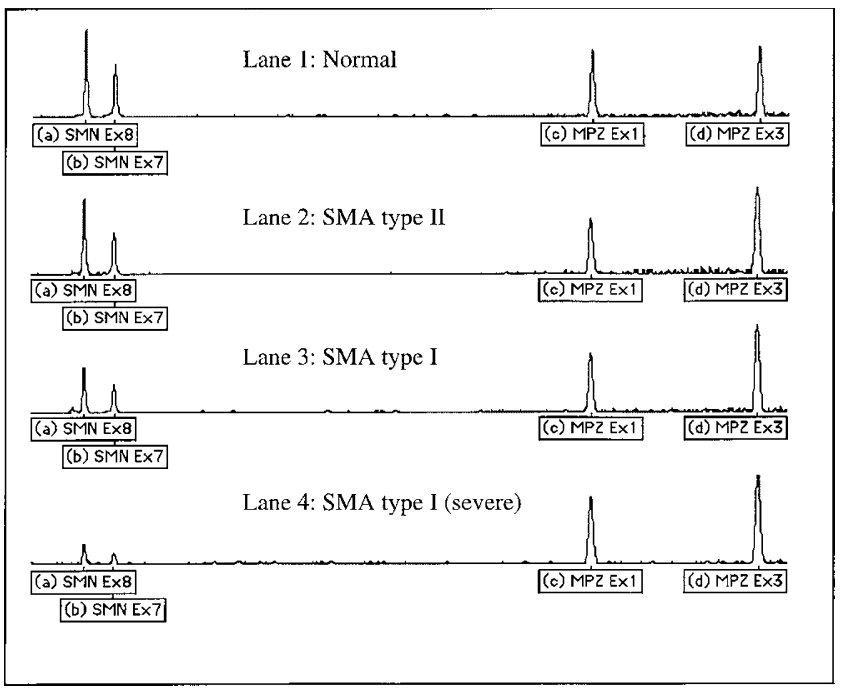

Figure 3 Fluorescent dosage assay of SMNC, showing reduced peak areas for SMN exons 7 and 8 associated with a more severe phenotype

A Ithough the correlation between the presence or absence of NAIP and the severity of phenotype is highly significant, the $(--+)$ genotype group contains a substantial number of SMA type I patients. The phenotypes in type I patients in whom NAIP was present or absent were therefore compared. The (- - ) group had a mean age at onset of 2.1 months $(n=61)$, and a mean age at death of 8.1 months $(n=44)$, whereas in the $(--+)$ group the mean age at onset was 2.0 months $(n=32)$ and mean age at death was 8.0 months $(n=20)$. Thus the NAIP deletions had no effect on the phenotype of type I patients.

The hypothesis that copy number of the SM N c gene influences the clinical phenotype was therefore investigated. A dosage assay was devised to measure the copy number of this gene in patients who had homozygous absence of the SM N t gene, but in whom the NA IP gene was present (group 2). These included 26 patients classified as SM A type I, 23 as type II and eight as type III. The type of data produced by this assay is shown in Figure 3, which clearly illustrates a reduced SMNC dosage in SMA type I. Lane 1 shows a control individual, with lanes 2-4 showing patients in increasing order of severity - SM A type II, type I and severe type I. It can be seen that the peak areas for SM N exons 7 and 8 are reduced in more severely affected patients.

The boxplot in Figure 4 shows mean SMN:MPZ ratios for each group of patients with the $(--+)$ genotype, and for the control group $(+++)$ of 27 unaffected individuals, with $95 \%$ and $99 \%$ confidence intervals. The median of the ratios reduces pro- gressively from type III to type I, and the ratios are substantially lower for SM A type I. The median and range for type III is similar to the controls, although the control values include one or more copies of SM N t which cannot be distinguished from SMNC in this assay.

The lowest ratio observed (0.11) was in a type I patient who was unusually severely affected, with reduced foetal movements apparent from 30 weeks of gestation, extreme hypotonia at birth, and death on day 4. Pairwise testing of dosage ratios between the four groups showed significant differences between all groups except between type III patients and controls ( $P<0.002$ in each case). Fitting a linear model with the subgroup as a factor was highly significant and accounted for $40 \%$ of the variance in the data. A nalysis of variance showed a highly significant effect of the subgroup on the values obtained, with $P<0.0001$. The SM NC gene dosage is plotted as a function of age of onset and survival in Figure 5 and Figure 6. These figures indicate that high SMN:MPZ ratios are significantly associated with both a later age of onset of SMA and a longer survival time. The age of onset increases approximately linearly with gene dosage $\left(r^{2}=0.48, P<0.0001\right)$. For survival, there was a sharp cut-off at a gene dosage ratio of 0.4 , and a ratio lower than this is associated with significantly reduced survival, having an effect similar to that of patients in the (- - ) genotypic group.

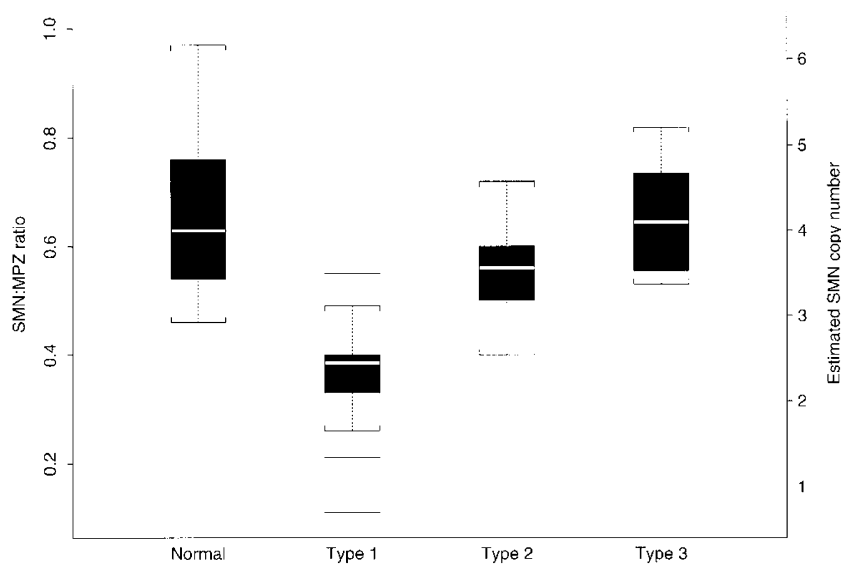

Figure 4 Boxplot of SM N c ratios vs SM A types I, II and III (all of these patients lack SMNt). The median ratio is represented by a white horizontal line. The $95 \%$ confidence intervals are represented by the solid box, with the $99 \%$ confidence intervals being represented by the vertical dotted lines. O utliers are represented by the horizontal lines above and below the boxes. (Note that in the controls the dosage ratio reflects the copy number of both the SM NC and SM N t genes) 


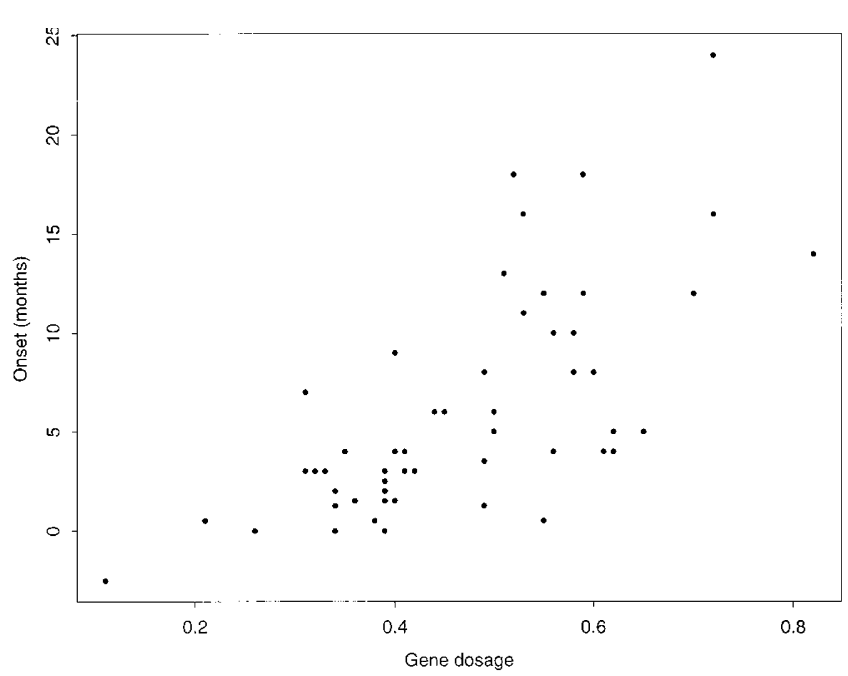

Figure 5 O nset by gene dosage, showing a positive correlation between gene dosage ratio and age at onset of disease

A wide range of SM N:MPZ ratios was seen in each group, and it is not possible to assess exact copy numbers from these ratios. However, if one assumes that the median dosage ratio observed in the normal control group (0.63) is most likely to represent four copies of SM N C + SM Nt genes, an extrapolation could be made from this to estimate SM N copy number in SMA patients (see right-hand axis in Figure 4). This would give the following results:

- Normal: median copy number 4, range 2.9-6.2 copies (SM Nt and SM NC);

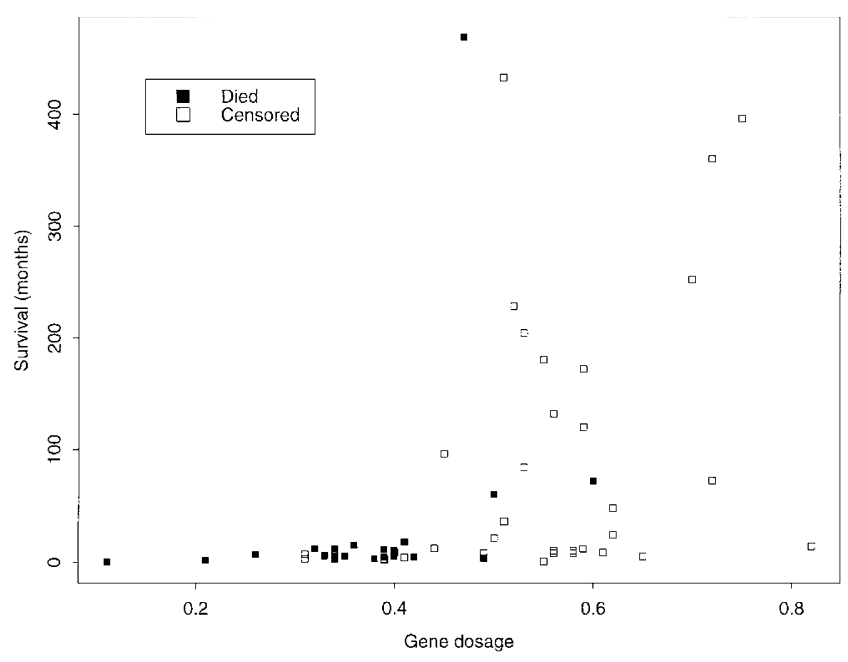

Figure 6 Survival by gene dosage, showing a positive correlation between gene dosage ratio and survival time. A gene dosage ratio less than 0.4 is strongly associated with poor survival time
- Type III: median copy number 4.1 , range 3.4-5.2 copies (SM N c only);

- Type II: median copy number 3.6 , range 2.5-4.6 copies (SM N c only);

- Type I: median copy number 2.4 , range $0.7-3.5$ copies (SM N c only).

The reproducibility of the assay was examined by dosage analysis of one SMA type I patient on six different occasions. This produced an estimated copy number of 2-2.7 copies. Thus variation in experimental conditions could account for the non-integral values of copy number observed in some patients.

\section{Discussion}

It is now well established that the SM Nt gene is absent in a high proportion of SMA patients, and that deletions of the NAIP gene are found in the majority of type I patients, but only rarely in types II and III. 5,16-19,30 This correlation has been extended by assessing the clinical phenotype of the four genotypic groups defined by the SM Nt and NAIP PCR assays in terms of the measurable clinical parameters, and subjecting the data to formal statistical analysis. It is apparent that the (-- -) genotype is associated with an earlier age of onset of the disease and survival relative to the $(--+)$ genotype, and that these differences are statistically highly significant. However, the fact that there was no difference in age of onset or survival in type I patients with the (---) and $(--+)$ genotype indicates that it is not the absence of the NAIP gene that determines the clinical phenotype.

The hypothesis that the extensive variation in the clinical phenotype of patients with apparently identical genotypes as defined by the SMN and NAIP PCR assays results from differences in copy number of the SM N c gene was then investigated. Fifty-seven patients from genotype group 2 (homozygous absence of the SMNt gene with NAIP present) were typed with a fluorescent dosage assay which measures the relative number of copies of the SM N C gene. Highly significant differences in SMNC copy number were observed among all three SM A types (Figure 4), and only type III patients were not significantly different from controls. The correlation of SM N c copy number and phenotype was also highly significant when analysed with respect to age of onset and survival (Figure 5 and Figure6). Dosage values of less than 0.4 , estimated to be 
equivalent to 1-2 copies of SM N c, were associated with particularly early onset and poor survival. The most severe phenotype in our series was associated with a dosage ratio of 0.11 , which is likely to reflect a single copy of the SMNC gene. Since no individuals with absence of both SMNt and SMNC genes have been reported, it is likely that disruption of both genes would constitute a lethal mutation. The fact that type III patients with the $(--+)$ genotype had SM N dosage values similar to those of controls can be explained by assuming that while the total number of SM N genes of any type is similar in these two groups, normal individuals (who have at least one copy of the fully functional SMNt) have fewer copies of SMNC than these type III patients. If the model of copy number proposed above is valid, normal individuals could have as many as 6 copies of SMNt and SMNC genes, in agreement with the study of Velasco et al. ${ }^{16} \mathrm{~A}$ Ithough the correlation between the SM N c dosage and measurable clinical parameters of clinical severity was not absolute, the results do indicate that in the absence of SM N t, the phenotype is strongly related to SM N c copy number.

This analysis is consistent with the recent study of L efebvre et al, who used antibody staining to show both that the SM N c gene is expressed in SM A patients, and that SM N protein levels are correlated with the severity of the disorder. ${ }^{23}$ It also supports the earlier study of Velasco et al, ${ }^{16}$ who demonstrated an increased SM N Cl SMNt ratio in the parents of type II and type III patients relative to the parents of type I patients. A dditionally, M CA ndrew et $\mathrm{al}^{31}$ have recently used a quantitative PCR assay to show that an asymptomatic SM A carrier with no copies of the SM N t gene had four copies of the SM N c gene, in keeping with the findings of this study. However, three haploidentical siblings from SM A families with discordant phenotypes showed no difference in SMNt or SM NC copy numbers. The correlation between phenotype and protein level is also not absolute. Coovert et $\mathrm{al}^{24}$ found that in five type I patients the mean SMN protein level was $17 \%$ of normal (range 9-27\%), whereas in eight type II patients it was $28 \%$ (range $15-41 \%$ ). H owever, there was a very strong correlation between the nuclear staining pattern as reflected in the number of gems from 100 nuclei, which was 5 in type I and 20 in type II patients. Thus, all SMNC genes are not likely to be functionally equivalent. ${ }^{24}$

This study also addresses the question of the molecular basis of the absence of the SM N t gene in SM A type
I patients in whom the NAIP gene is present. B urghes ${ }^{32}$ points out that whereas the (-- ) genotype in SMA type $I$ is likely to reflect a deletion and the $(--+)$ genotype in types II and III a conversion, the $(--+)$ genotype in SM A type I could be either a deletion or a conversion. The data in Figure 4 show that most type I patients with the $(--+)$ genotype have approximately two copies of SMNC, whereas the type II and III patients have 3-5 copies. This suggests that the SM Nt gene is removed by a deletion in the majority of $(--+)$ type I patients.

In conclusion, this study demonstrates that although there is a highly significant correlation between the presence or absence of the NAIP gene and the severity of the clinical phenotype in SM A, its presence does not influence age of onset or survival in a subgroup of severely affected patients. This is consistent with the fact that small mutations within the SM Nt gene do not disrupt NAIP, but may still give rise to a severe phenotype. $^{7-9}$ The study also confirms the strong correlation between SM NC copy number and phenotype in patients lacking an SM Nt gene, and demonstrates the value of assessing clinical phenotype in terms of quantifiable variables which can be subjected to formal statistical analysis. This is particularly relevant in the context of the difficulties that may be encountered in classification of SMA patients into defined subgroups. ${ }^{33,34}$

\section{Acknowledgements}

We would like to thank the Muscular Dystrophy Group of $G$ reat B ritain and Northern Ireland, the Jennifer Trust for Spinal M uscular A trophy and the Nathalie-J o B irthday Walk for their support. We are grateful to Lydie Burglen and J udith Melki for providing us with unpublished sequence information. Thanks are also due to M r SC Y au for helpful discussions and advice, and to John M artindale and $E$ lizabeth $M$ anners for editorial assistance.

\section{References}

1 Brzustowicz LM, Lehner T, Castilla LH et al: Genetic mapping of chronic childhood-onset spinal muscular atrophy to chromosome 5q11.2-13.3. Nature 1990; 344: 540-541.

2 Gilliam TC, B rzustowicz LM, Castilla LH et al: Genetic homogeneity between acute and chronic forms of spinal muscular atrophy. Nature 1990; 345: 823-825.

3 Melki J, Sheth P, A bdelhak S et al and the French Spinal M uscular A trophy Investigators: M apping of acute (type I) spinal muscular atrophy to chromosome 5q12-q14. L ancet 1990; 336: 271-273. 
4 Melki J, Lefebvre S, Burglen L et al: De novo and inherited deletions of the $5 q 13$ region in spinal muscular atrophies. Science 1994; 264: 1474-1476.

5 Lefebvre S, Burglen L, Reboullet $S$ et al: Identification and characterization of a spinal muscular atrophy-determining gene. Cell 1995; 80: 155-165.

6 R oy N, M ahadevan MS, MCLean M et al: The gene for neuronal apoptosis inhibitory protein is partially deleted in individuals with spinal muscular atrophy. Cell 1995; 80: 167-178.

7 Bussaglia E, Clermont O, Tizzano E et al: A frame-shift deletion in the survival motor neuron gene in Spanish spinal muscular atrophy patients. Nat Genet 1995; 11: 335-337.

8 Williams Parsons D, M CA ndrew PE, M onani U R, M endell $J R$, B urghes A H M , Prior TW: A n 11 base pair duplication in exon 6 of the SMN gene produces a type I spinal muscular atrophy (SM A) phenotype: further evidence for SM N as the primary SM A -determining gene. $\mathrm{Hum} \mathrm{Mol}$ G enet 1996; 5: 1727-1732.

9 Brahe C, Clermont O, Zappata S, Tiziano F, M elki J, Neri $G$ : Frameshift mutation in the survival motor neuron gene in a severe case of SM A type I. H um M ol G enet 1996; 5: 1971-1976.

10 Talbot $K$, Ponting $C P$, Theodosiou $M$ et al: $M$ issense mutation clustering in the survival motor neuron gene: a role for a conserved tyrosine and glycine rich region of the protein in RNA metabolism? Hum Mol Genet 1997; 6: 497-500.

$11 \mathrm{H}$ ahnen $\mathrm{E}$, Schonling J, Rudnik-Schoneborn S, R aschke $H$, Z erres K, Wirth B: M issense mutations in exon 6 of the survival motor neuron gene in patients with spinal muscular atrophy (SMA). Hum Mol Genet 1997; 6: 821-825.

12 Wirth $B, H$ ahnen $E$, Morgan $K$ et al: A llelic association and deletions in autosomal recessive proximal spinal muscular atrophy: association of marker genotypes with disease severity and candidate cDNA s. Hum Mol G enet 1995; 4: 1273-1284.

13 Cobben J M, van der Steege G, G rootscholten P, de V isser $M$, Scheffer $H$, Buys CHCM: Deletions of the survival motor neuron gene in unaffected siblings of patients with spinal muscular atrophy. Am J Hum Genet 1995; 57: 805-808.

$14 \mathrm{H}$ ahnen $\mathrm{E}$, Forkert $\mathrm{R}, \mathrm{M}$ arke $\mathrm{C}$ et al: $\mathrm{M}$ olecular analysis of candidate genes on chromosome $5 q 13$ in autosomal recessive spinal muscular atrophy: evidence of homozygous deletions of the SM N gene in unaffected individuals. $\mathrm{H}$ um M ol G enet 1995; 4: 1927-1933.

15 Wang $\mathrm{CH}, \mathrm{Xu}$ J, Carter TA et al: Characterization of

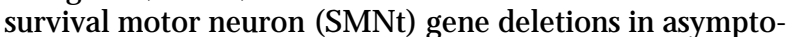
matic carriers of spinal muscular atrophy. $\mathrm{H}$ um $\mathrm{M}$ ol $\mathrm{G}$ enet 1996; 5: 359-365.

16 Velasco E, Valero C, Valero A, Moreno F, HernandezChico C: M olecular analysis of the SM N and NAIP genes in Spanish spinal muscular atrophy (SMA) patients and correlation between number of copies of CBCD 541 and SM A phenotype. Hum Mol G enet 1996; 5: 257-263.

17 Burlet $P, B$ urglen $L$, Clermont $O$ et al: $L$ arge scale deletions of the $5 q 13$ region are specific to WerdnigH offmann disease. J M ed G enet 1996; 33: 281-283.
18 Samilchuk E, D 'Souza B, B astaki L, A I-A wadi S: D eletion analysis of the SM N and NA IP genes in Kuwaiti patients with spinal muscular atrophy. $\mathrm{Hum}$ Genet 1996; 98: 524-527.

19 Somerville MJ, H unter A GW, A ubry HL, Korneluk RG, $M$ ack enzie $A E$, Surh LC: Clinical application of the molecular diagnosis of spinal muscular atrophy: deletions of neuronal apoptosis inhibitor protein and survival motor neuron genes. A m J M ed G enet 1997; 69: 159-165.

20 Van der Steege G, G rootscholten PM, Cobben J M et al: A pparent gene conversions involving the SM N gene in the region of the spinal muscular atrophy locus on chromosome 5. A m J H um G enet 1996; 59: 834-838.

$21 \mathrm{H}$ ahnen $\mathrm{E}$, Schonling J, R udnik-Schoneborn S, Zerres K, Wirth B: H ybrid survival motor neuron genes in patients with autosomal recessive spinal muscular atrophy: new insights into molecular mechanisms responsible for the disease. A m J H um G enet 1996; 59: 1057-1065.

22 Campbell L, Potter A, I gnatius J, D ubowitz V, D avies K: $G$ enomic variation and gene conversion in spinal muscular atrophy: implications for disease process and clinical phenotype. A m J H um G enet 1997; 61: 40-50.

23 Lefebvre S, Burlet $\mathrm{P}$, Liu Q et al: Correlation between severity and SM N protein level in spinal muscular atrophy. $N$ at $\mathrm{G}$ enet 1997; 16: 265-269.

24 Coovert DD, Le TT, MCA ndrew PE et al: The survival motor neuron protein in spinal muscular atrophy. Hum Mol G enet 1997; 6: 1205-1214.

25 M unsat TL: International SMA collaboration workshop report. Neuromuscul D isord 1991; 1: 81.

26 M unsat TL, D avies KE: M eeting R eport - International SMA consortium meeting report. Neuromuscul Disord 1992; 2: 423-428.

27 Van der Steege G, G rootscholten PM , van der V lies P et al: PCR -based DNA test to confirm the clinical diagnosis of autosomal recessive spinal muscular atrophy. $L$ ancet 1995; 345: 985-986.

28 Yau SC, Bobrow M, Mathew CG, A bbs SJ: A ccurate diagnosis of carriers of deletions and duplications in D uchenne/B ecker muscular dystrophy by fluorescent dosage analysis. J Med G enet 1996; 33: 550-558.

29 Hayasaka $\mathrm{K}, \mathrm{H}$ imoro $\mathrm{M}$, Wang $\mathrm{Y}$ et al: Structure and chromosomal localization of the gene encoding the human myelin protein zero (MPZ). Genomics 1993; 17: 755-758.

30 R odrigues NR, O wen N, Talbot K, I gnatius J, D ubowitz V, $D$ avies KE: D eletions in the survival motor neuron gene on $5 q 13$ in autosomal recessive spinal muscular atrophy. $\mathrm{H}$ um Mol G enet 1995; 4: 631-634.

31 M CA ndrew PE, Parsons DW, Simard LR et al: Identification of proximal spinal muscular atrophy carriers and patients by analysis of SMNt and SMNC gene copy number. A m J H um G enet 1997; 60: 1411-1422.

32 Burghes A H M : When is a deletion not a deletion? When it is converted. A m J H um G enet 1997; 61: 9-15.

33 Dubowitz $\mathrm{V}$ : Chaos in classification of the spinal muscular atrophies of childhood. Neuromuscul Disord 1991; 1: 77-80.

34 Dubowitz V: Chaos in classification of SM A : a possible resolution. Neuromuscul Disord 1995; 5: 3-5. 